\title{
Use of a Transarticular Calcaneo-Tibial Locking Plate for Temporary Immobilization of the Tarsocrural Joint following Surgical Repair of Common Calcaneal Tendon Rupture in Eight Dogs
}

\author{
Alessandro Boero Baroncelli ${ }^{1,2}$ Francesca Chiara Ferrero ${ }^{2,3}$ Luca Omodeo ${ }^{4}$ Mattia Sarotti ${ }^{5}$ \\ Bart Verdonck $^{6}$ Bruno Peirone ${ }^{7}$ Lisa Adele Piras ${ }^{7}$ \\ ${ }^{1}$ Centro Veterinario Imperiese, Imperia, Italy \\ ${ }^{2}$ Clinica Albese per Animali da Compagnia, Alba, Cuneo, Italy \\ ${ }^{3}$ Clinica Veterinaria Serravalle, Serravalle Scrivia, Italy \\ ${ }^{4}$ Anubi Ospedale Veterinario, Moncalieri, Italy \\ ${ }^{5}$ Centro Veterinario Fossanese, Fossano, Italy \\ ${ }^{6}$ Huisdierchirurgie-Verdonck, Boechout, Belgium \\ ${ }^{7}$ Dipartimento di Scienze Veterinarie, Università di Torino, \\ Grugliasco (To), Italy \\ Vet Comp Orthop Traumatol 2021;34:359-366. \\ Address for correspondence Lisa Adele Piras, DVM, PhD, \\ Dipartimento di Scienze Veterinarie, Università di Torino, Turin, \\ Largo Paolo Braccini 2-5, Grugliasco 10095, Italy \\ (e-mail: lisa.piras@unito.it).
}

\begin{abstract}
Keywords

- tendon repair

- locking plates

- tendon healing

- dogs

- common calcaneal tendon

Objective The purpose of this clinical communication is to report the management of common calcaneal tendon rupture in dogs using a transarticular calcaneo-tibial locking plate as a method of temporary immobilization of the tarsocrural joint to support primary repair of the common calcaneal tendon.

Study Design Dogs presented with partial or complete common calcaneal tendon rupture treated with primary tendon repair supported by the transarticular calcaneotibial locking plate technique from July 2016 to December 2019 were included. The tarsus was placed at a standing angle of 150 degrees and the pre-contoured Fixin locking ' 'L' or ' $T$ ' plate was medially applied with two locking screws inserted in the calcaneus and two screws inserted in the distal tibia. Tendon healing judgement was mostly based on clinical palpation and ultrasound examination was not performed in all the cases. Plates were removed in all dogs 6 weeks postoperatively.

Results All tendons healed and no complications at the level of the tenorrhaphy were noted in the postoperative period. All dogs returned to hunting activity with full function of the affected limb. No major complications requiring revision surgery were observed. Minor complications occurred in two cases; osteomyelitis and screw pull-out. Conclusion The use of a transarticular calcaneo-tibial locking plate for temporary immobilization of the tarsocrural joint after surgical repair of common calcaneal tendon rupture appears to be an effective technique with comparable results to other reported techniques.
\end{abstract}

received

June 17, 2020

accepted after revision

April 3, 2021

published online

June 11, 2021 


\section{Introduction}

The common calcaneal tendon attaches to the calcaneal tuberosity in domestic mammals. It includes the superficial digital flexor tendon, gastrocnemius and common tendons formed from the biceps femoris, gracilis and semitendinosus muscles, and is bound down by the lamina propria of deep sheath of the crural fascia. ${ }^{1}$ Disruption of one or more components is a disabling injury in dogs that compromise the ability to maintain the extension of the tarsocrural joint. Common calcaneal tendon injuries are reported to occur as a result of a direct trauma or secondary to degenerative conditions. $^{2,3}$ In dogs, the injury is classified according to the duration of the lesion as acute or chronic, and the amount of tendon involvement as partial or complete. Partial rupture, involving the gastrocnemius muscle tendon, the tendon of the biceps femoris, semitendinosus and gracilis muscles with an intact superficial digital flexor tendon, is reported to be more common in dogs. ${ }^{3}$ Dogs affected by degenerative insertional lesions present elongation of both gastrocnemius and the semitendinosus and gracilis tendons, with an intact superficial digital flexor tendon. Depending upon the degree and duration of the injury, they usually present with a typical clawing posture. ${ }^{4}$ Surgical repair is the generally accepted recommendation for the effective return to function. ${ }^{4}$ Primary repair of a mid-body laceration of the calcaneal tendon typically involves suturing the severed ends directly together, whereas insertional injuries often require bone tunnels in the calcaneus. Different suture patterns are reported; of these, the three-loop pulley pattern is generally favoured for the reattachment of round or semi-round tendons to bone. In addition to suturing, adjunctive bridging of the tendon has been described with a fascia lata autograft, semitendinosus flap, polypropylene mesh, porcine intestinal submucosa, epitendinous sutures, fascial grafts, tendon transfers and the use of biological and artificial implants. ${ }^{2,5-15}$ Several methods have been described to avoid overstress of the anastomosis and to allow isometric muscle contraction during the early postoperative period. Use of transarticular external fixation, ${ }^{4,6,7,16}$ calcaneo-tibial screw, ${ }^{4,8,16}$ cast application, ${ }^{17}$ a single ring transarticular fixator ${ }^{18}$ and a custom orthotic boot ${ }^{19}$ has been reported.

The purpose of this clinical communication is to report the management of common calcaneal tendon rupture in dogs using a transarticular calcaneo-tibial locking plate technique as a method of temporary immobilization of the tarsocrural joint to support primary repair of common calcaneal tendon injuries. It was our objective to determine whether this technique would provide adequate immobilization of the tarsocrural joint during the initial phase of tendon healing with minimal morbidity and a low incidence of complications.

\section{Materials and Methods}

All dogs presented with partial or complete common calcaneal tendon rupture treated with primary tendon repair supported by the transarticular calcaneo-tibial locking plate

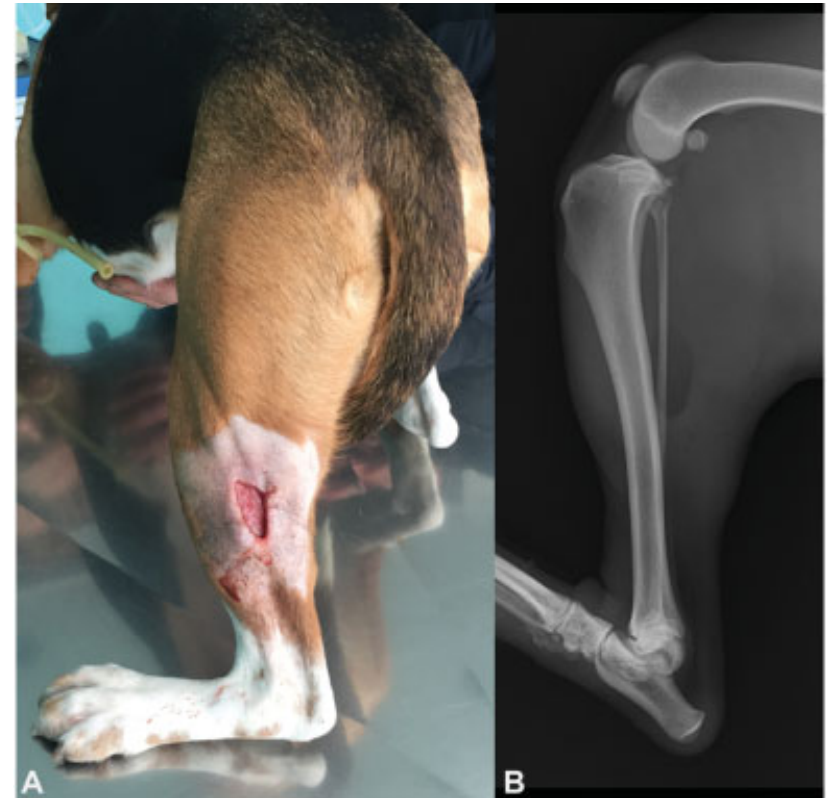

Fig. 1 (A) Clinical presentation of a dog with complete common calcaneal tendon lesion and plantigrade posture; (B) Mediolateral radiographic view with hyperflexion of the hock joint.

technique from July 2016 to December 2019 were included. The diagnosis of partial or complete disruption to the common calcaneal tendon mechanism was based on orthopaedic examination and radiographic findings ( - Fig. 1). Only cases with a complete medical record, orthopaedic examination performed by the surgeon, preoperative, immediate postoperative and follow-up radiographs performed at 4 weeks and after plate removal were included in the study. Dogs with concomitant neurologic or orthopaedic pathologies without a complete medical record were excluded from the study.

The following parameters were recorded for all patients: breed, age (months), body weight $(\mathrm{kg})$, duration of lameness, localization (body, tendon insertion on the calcaneus, musculotendinous junction), traumatic or degenerative, partial or complete and the type of plate used.

Dogs were premedicated with fentanyl ( $4 \mu \mathrm{g} / \mathrm{kg}$ intravenous [IV]; Fentanest, Actavis Italy S.p.A, Nerviano, Italy), morphine $(0.15 \mathrm{mg} / \mathrm{kg}$ intramuscular [IM]; Morfina Cloridrato, Molteni, Italy) and acepromazine $(0.02 \mathrm{mg} / \mathrm{kg} \mathrm{IM}$; Prequillan, Fatro SpA, Ozzano Emilia, Italy).

Anaesthesia was induced with propofol (3 mg/kg IV; PropoVet; Ecuphar Italia S.p.A, Milano, Italy) and maintained with a mixture of isoflurane (IsoFlo: Zoetis Italia S.r.l, Roma, Italy) in $2 \%$ oxygen after endotracheal intubation. A constant rate infusion of fentanyl $(10 \mu \mathrm{g} / \mathrm{kg} / \mathrm{h}$ IV; Fentanest, Actavis Italy S.p.A, Nerviano, Italy) provided analgesia, and cefazolin (20 mg/kg, IV; Cefazolina Dorom, Teva Pharma Italia, Milano, Italy) was administered 1 hour before surgery and repeated 120 minutes later. The anaesthetized dogs were positioned in dorsal recumbency, and the limbs were aseptically prepared in a hanging position. A caudomedial surgical approach to the tarsocrural joint was performed. The retinaculum was incised caudal to the plantar nerve, the flexor hallucis longus and the medial saphenous artery and vein (-Fig. 2). 


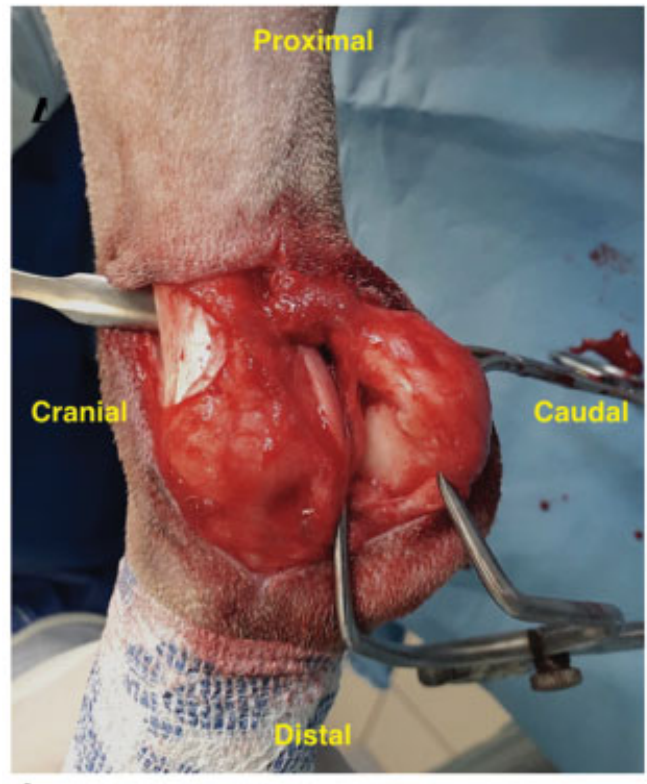

A

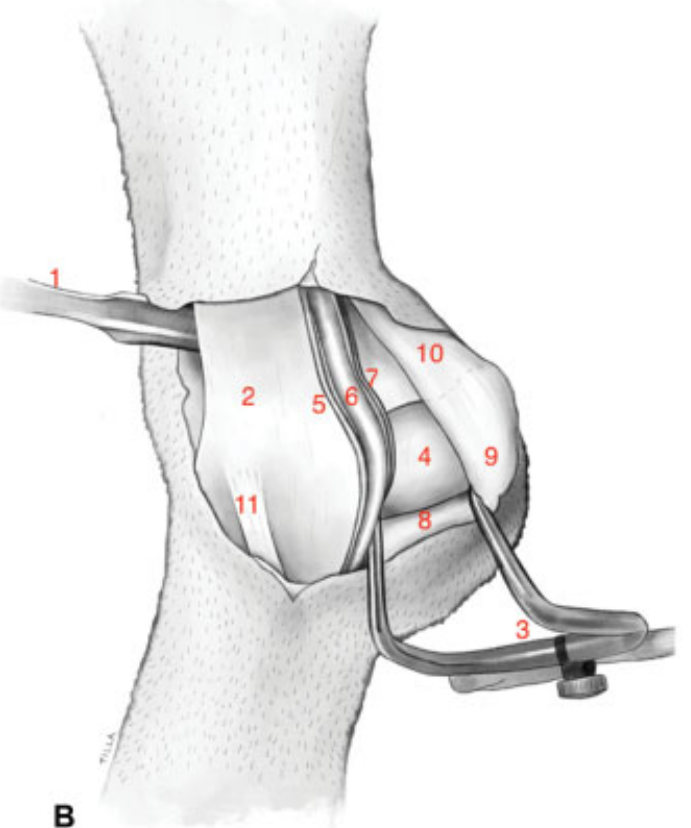

B

Fig. 2 Intraoperative caudomedial view of the surgical approach with the dog in dorsal recumbency. (A) Schematic description of the regional anatomy. (B) A small Hohmann retractor (1) is retracting the skin and the subcutaneous tissue cranially to expose the distal tibia. (2) After the incision of the distal retinaculum, a Gelpi retractor (3) is applied to expose the calcaneus (4) retracting cranially the plantar nerve (5), the flexor hallucis longus (6) and the medial saphenous artery and vein (7) and caudally, the tendon of the superficial digital flexor muscle (8) without opening the subtendinous synovial bursae; (9) common calcaneal tendon; (10) medial collateral ligament (11).

Immobilization of the tarsocrural joint in extension was achieved by medial application of a locking bone plate (FIXIN, Intrauma s.r.l., Rivoli, TO).$^{20}$ Plate benders ${ }^{20}$ designed for this system were used to avoid deformation of the titanium bushings during contouring of the stainless-steel implant. The tarsus was placed at a standing angle of 150 degrees. The pre-contoured locking plate was medially applied with two locking screws inserted in the calcaneus and two screws inserted in the distal tibia. A 'L' or ' $T$ ' plate was selected according to the surgeon preference. In both cases, the plate was positioned with the shorter side over the calcaneus (-Fig. 3). A caudolateral approach was performed in cases of proximal lesion to suture the musculotendinous junction, while all the other lesions were treated through a proximal extension of the caudomedial approach. In traumatic cases, primary repair of individual tendons was performed. All of the tendons were repaired with a modified three-loop pulley or locking loop suture pattern, ${ }^{3,21}$ using non absorbable 3-metric monofilament nylon suture or 3metric monofilament polypropylene suture (Prolene, Ethicon Ltd, Edinburgh, UK). All the knots were tied with a double throw followed by three single throws. All the sutures were
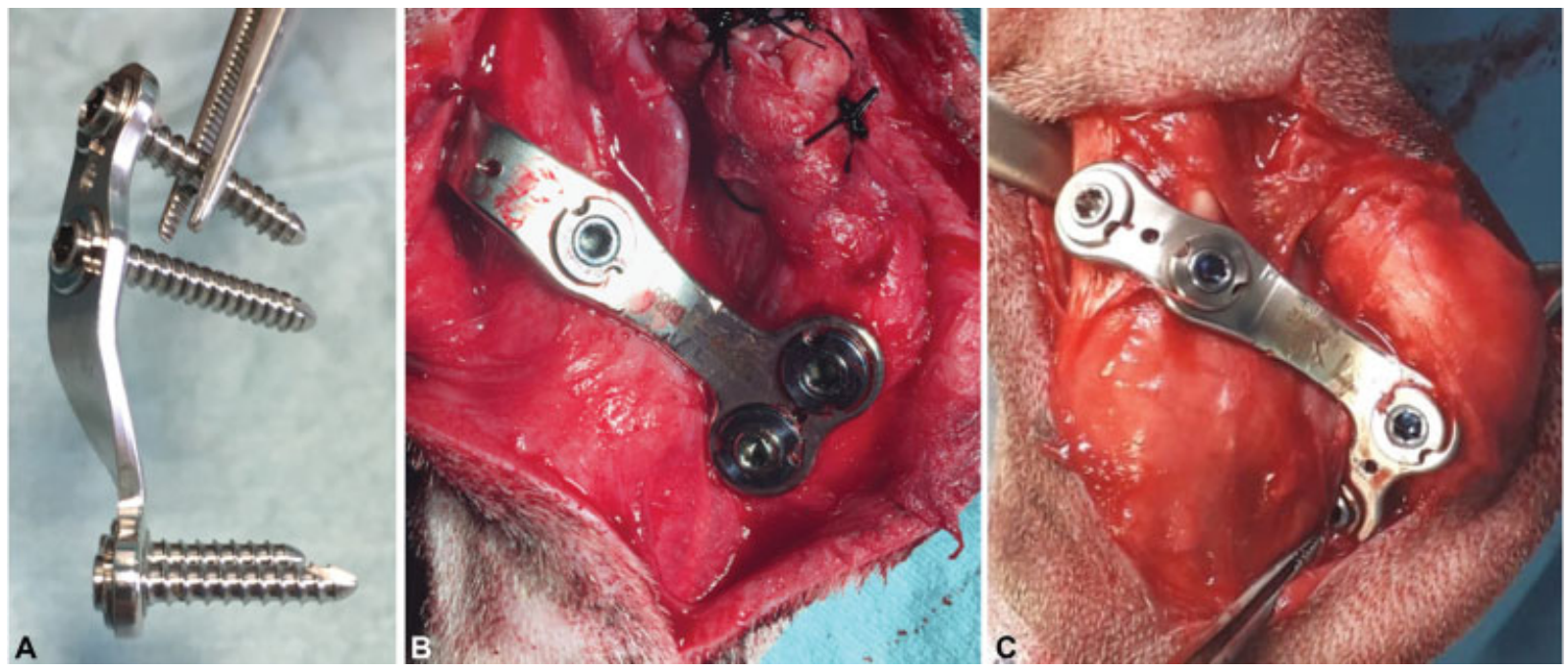

Fig. 3 (A) Pre-contoured locking plate with the locking screws inserted. (B) Intraoperative view of medial locking plate application with two locking screws in the calcaneus and two in the distal tibia; (B) 'T' plate; (C) 'L' plate. 

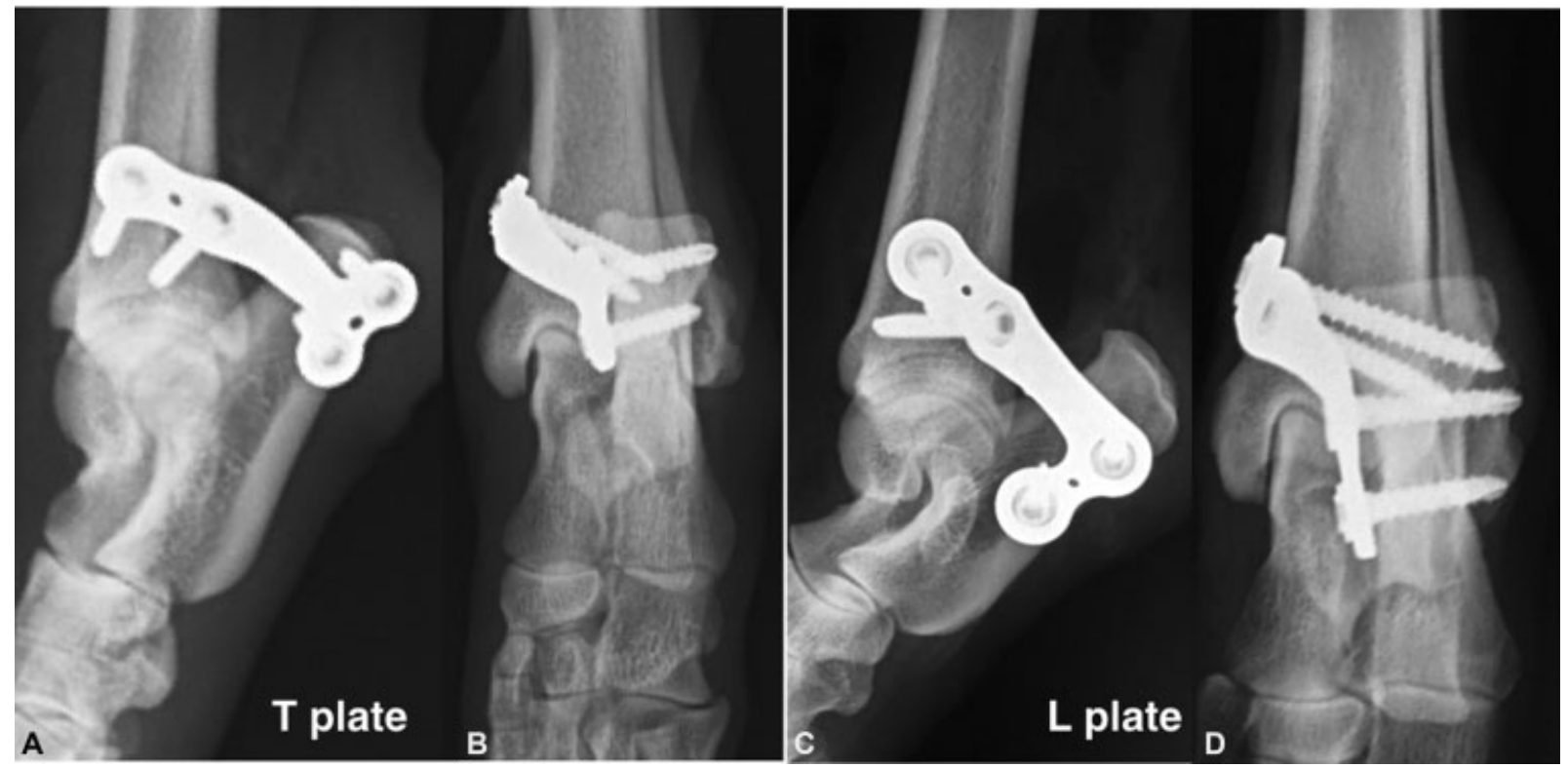

Fig. 4 Postoperative radiographs of medial 'T' plate (A, B) and 'L' plate (C, D) application.

tightened until the tendon ends were anatomically apposed and there was no slack within the suture. In degenerative cases, the chronic avulsion injury of the common calcaneal tendon was treated using sutures passed through bone tunnels. A $1.5 \mathrm{~mm}$ transverse bone tunnel was drilled through the lateral process of the calcaneal tuberosity just distal to the insertion of the gastrocnemius tendon to allow the suture to be anchored to the calcaneus. In the tendon, the far bite was taken $15 \mathrm{~mm}$, the middle bite $10 \mathrm{~mm}$ and the near bite $5 \mathrm{~mm}$ from the tendon bone junction. Each bite was placed to create three loops, each at a 60 degrees angle from the other loops. ${ }^{3}$ If contamination of the surgical site was suspected due to a traumatic injury, bacterial culture and susceptibility test were performed. The surgical wound was routinely flushed with saline without antibiotic medications. Reconstruction of the retinaculum after plate application was performed in a simple interrupted pattern, using 3.0 metric polydioxanone monofilament suture (PDS II, Ethicon Ltd, Edinburgh, UK) over the plate. The fascia and subcutis were closed in a simple interrupted pattern, using 3.0 metric polydioxanone (PDS II, Ethicon Ltd, Edinburgh, UK) monofilament suture. The skin was closed in a simple interrupted pattern. No cast was applied postoperatively. Postoperative orthogonal radiographs were obtained. All dogs were treated with meloxicam $(0.1 \mathrm{mg} / \mathrm{kg}$ orally, q12h; Meloxidolor, Dechra Veterinary Products S.r.l, Torino, Italy) for 7 days and cephalexin $(22 \mathrm{mg} / \mathrm{kg}$ orally, q8h; Icfvet, I.C.F. Industria Chimica Fine S.r.l, Cremona, Italy) for 7 days. When the result of bacterial culture was available, positive dogs were treated with antibiotic medications according to the sensitivity. All the dogs were restricted to short lead walks during the postoperative phase (average 10 to 15 minutes three times a day).

Patient outcome was evaluated based on the information obtained from surgeon's clinical evaluation, follow-up radiographs and long-term telephone follow-up. Time to follow- up examination was recorded in days. Clinical re-examinations were planned at 1,4 and 6 weeks. The degree of lameness and postoperative complications were recorded. Lameness was graded by the surgeon on a scale from 0 (no lameness) to 5 (consistently non-weight-bearing). ${ }^{22}$

Complications were recorded and were classified as either major or minor. Major complications were defined as those requiring revision surgery, or plate or screw breakage even if the breakage was treated conservatively. ${ }^{4}$ Minor complications were defined as complications not requiring revision surgery which resolved with medical or conservative management. ${ }^{4,23}$

Radiographs of the affected tarsus were taken 4 weeks postoperatively ( $\boldsymbol{- \text { Fig. }} \mathbf{4}$ ) to assess the tarsal angle, implant related complications or fracture. If tendon healing was judged satisfactory by ultrasound evaluation or palpation, dogs were anaesthetized and surgically prepared as previously, for planned removal of the temporary plate at 6 weeks post immobilization. After plate removal, dogs were gradually encouraged to controlled loading with restricted activity for 4 weeks and were gradually returned to normal exercise over 4 to 6 weeks. Long-term evaluation was subjectively performed by the surgeon 1 year postoperatively or by telephone interview.

\section{Results}

The temporary transarticular calcaneo-tibial locking plate technique was used in nine dogs following common calcaneal tenorrhaphy. One dog died after 5 weeks due to acute renal failure secondary to an acute leishmaniasis infection and was excluded from the study (-Appendix Table A1, available in the online version). Included breeds were two Beagles, four mixed breed dogs, one Dobermann and a Belgian Malinois. Four out of eight dogs were hunting dogs. All dogs were males and ranged in age from 4 to 16 years. Their weights ranged from 8 to $32 \mathrm{~kg}$ with a mean of $23.5 \mathrm{~kg}$. 
All dogs had been presented with the complaint of hindlimb lameness. The duration of lameness ranged between 2 and 21 days in traumatic cases and between 1 and 6 months in degenerative cases. All dogs had swelling of the common calcaneal tendon and signs of pain upon manipulation of the tarsocrural joint and upon direct pressure of the tendon. Six cases were presented for a complete traumatic rupture of the common calcaneal tendon with a plantigrade stance. Traumatic tendon ruptures were midbody in $5 / 6$ cases and at the musculotendinous junction in 1 case. Two cases were presented for a partial degenerative lesion at the level of tendinous insertion on the calcaneus with hyperflexion of the digits. Additional significant orthopaedic or neurological abnormalities of the thoracic or pelvic limbs were not observed. Locking-loop suture tenorrhaphy $(n=4)$ or a modified three-loop pulley suture technique $(n=2)$ was performed. ${ }^{4}$ Non-absorbable suture passed through bone tunnels was used to reappose insertional avulsions in two dogs. Temporary tarsocrural immobilization in extension was performed using four different plate types: a $3.5 \mathrm{~mm}$ ' $T$ ' shaped plate (V3003, V3043), a 'L' shaped $3.5 \mathrm{~mm}$ plate (V3034) and a $2.5 \mathrm{~mm}$ cuttable plate (V2207). All plates were applied to the medial side of the tarsus and the plate-bone contact was visually assessed. Bicortical screw insertion was performed in all cases. No intraoperative complications occurred. Aerobic and anaerobic bacteria culture was performed in three cases and yielded a positive culture result in two cases. Enterobacter cloacae and Burkholderia cepacia were cultured from the surgical site, and based on the results of culture and sensitivity, the combination of amoxicillin and clavulanic acid $(20 \mathrm{mg} / \mathrm{kg}$, orally, q12h; Synulox, Pfizer, Rome, Italy) was administered. Reconstruction of the retinaculum after plate application was performed in a simple interrupted pattern, using 3.0 metric polydioxanone (PDS II, Ethicon Ltd, Edinburgh, UK) monofilament suture over the plate. In the case of troublesome suturing of the medial flexor digit superficial retinaculum, we used the two calcaneal locking screws to anchor the suture in position. There were no cases of superficial digital flexor tendon luxation in the postoperative period due to this procedure. ${ }^{24}$ All wounds, surgical and traumatic, were closed primarily and did not require additional bandages. In all cases, the immediate postoperative radiographs showed satisfactory hock extension, adequate implant position and correct screw length. Clinical evaluation after 7 days showed partial weight bearing of the affected limb in all dogs with II/V lameness in five cases and a III/V lameness in three cases. After 4 weeks, no lameness was present at the walk in two cases, I/V lameness was present in three cases, II/V lameness in two cases, while in one case III/V lameness was present with clinical signs of local infection. No major complications were observed in any dogs necessitating revision surgery. Minor complications occurred in two cases. Osteomyelitis of the calcaneus developed subsequent to surgery in one dog which had a preexisting infected wound, secondary to the dog's plantigrade stance. This was despite bacterial culture performed during surgery failing to yield bacterial growth. Radiographic follow-up at 4 weeks postoperatively showed bone resorption at the level of the tibial and calcaneal screw holes. A second bacterial culture confirmed Staphylococcus aureus infection and antibiotic therapy with clindamycin $(11 \mathrm{mg} / \mathrm{kg}$ orally, q12h; Zodon, Ceva Salute Animale S.p.a, Monza Brianza, Italy) was administered for 4 weeks. Implant removal was performed at 6 weeks postoperatively. Later radiographs showed increased bone density and reduced periosteal reaction on the calcaneal cortex. Screw pull-out from the tibia because of bushing loosening, without concurrent bone failure, was observed in another dog (-Fig. 5) at 6 weeks radiographic follow-up. Plates were removed in all cases after 6 weeks without intra- or postoperative complications (-Fig. 6). An initial reduction of range of motion in flexion was observed, but all dogs were free from lameness 12 weeks postoperatively. Long-term follow-up was clinically performed by the surgeon in 5/8 cases and tarsocrural function was judged subjectively satisfactory. All owners were
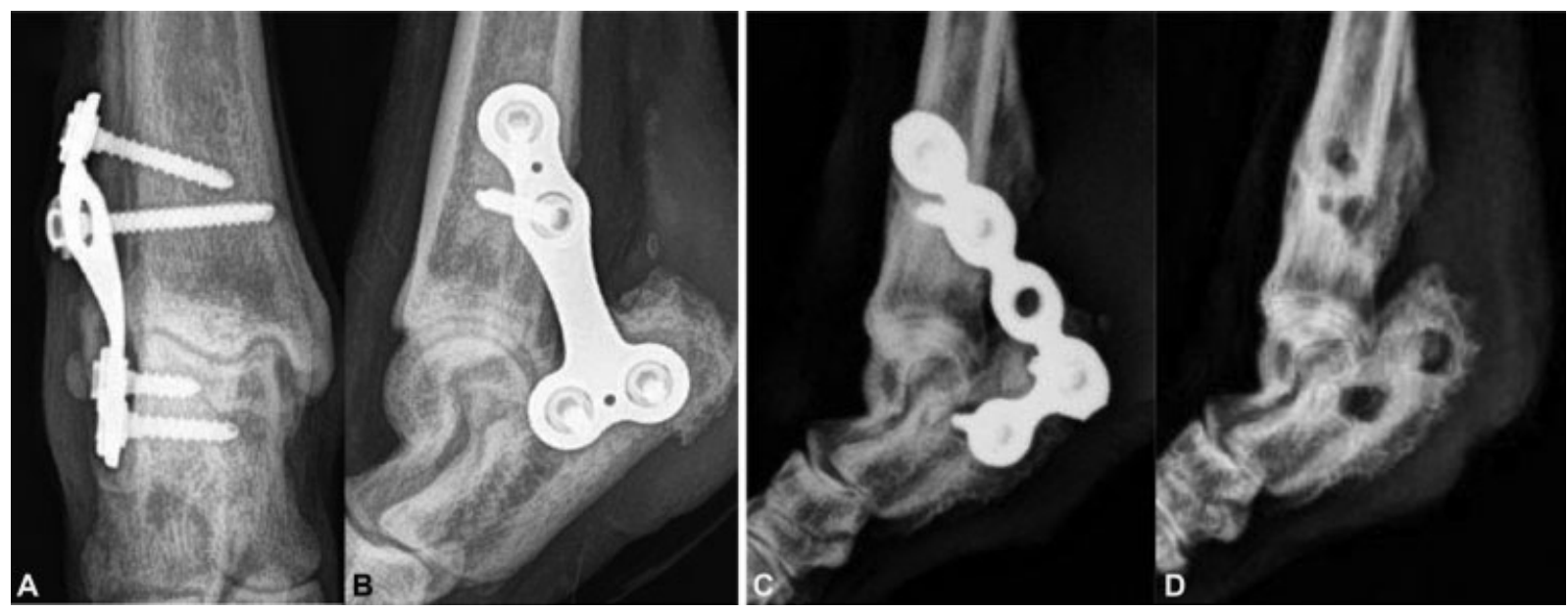

Fig. 5 Complications: Radiographic follow-up at 4 weeks (A, B) showing screw pull-out in case 7 (bacterial culture had not yielded bacterial growth). Severe case of bacterial osteomyelitis before (C) and after implant removal (D) at 6 weeks (bacterial culture confirmed Staphylococcus aureus infection) in case 1. 


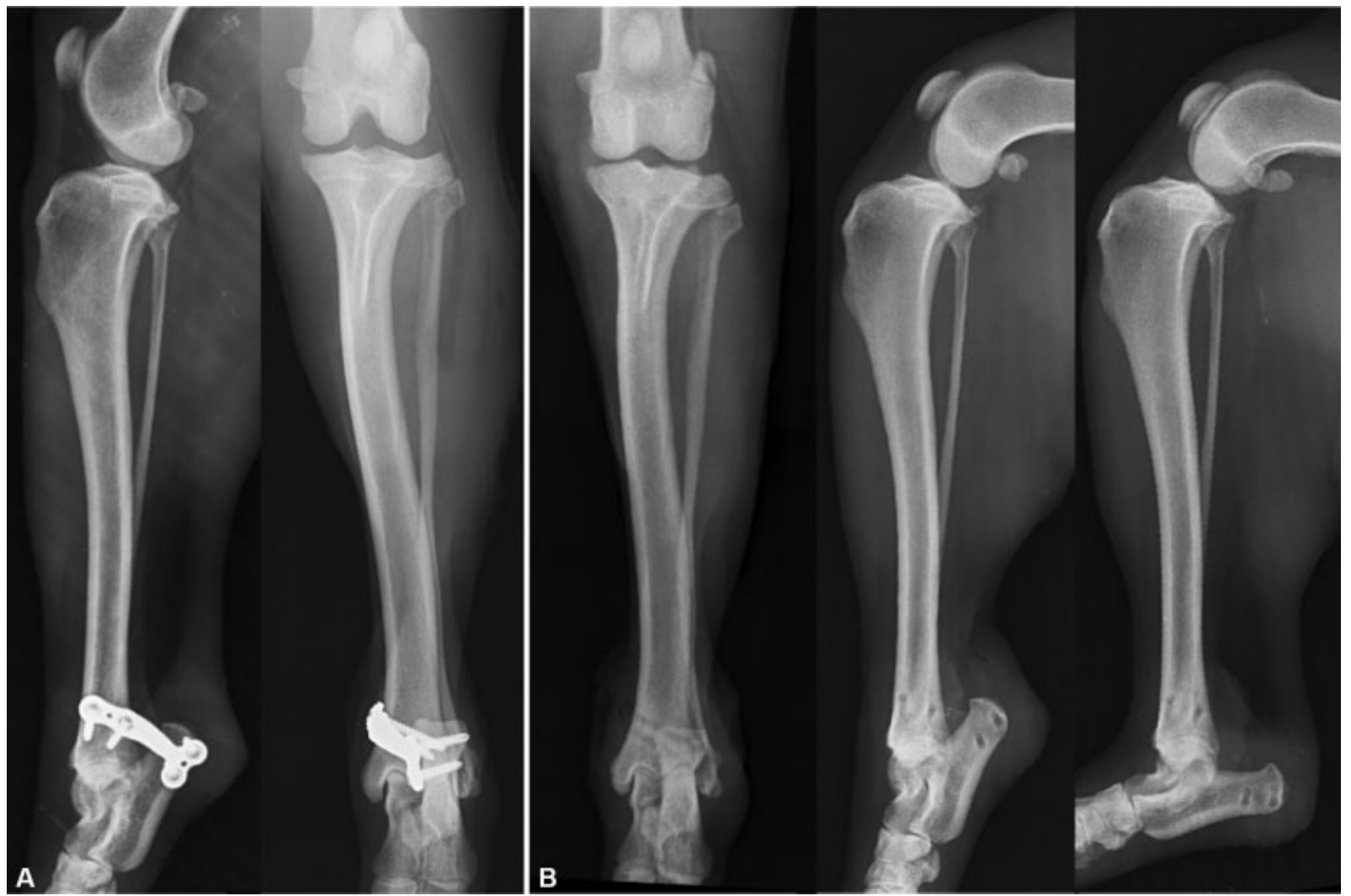

Fig. 6 Radiographic follow-up at 4 weeks (A) and at 6 weeks after implant removal (B).

available for follow-up contact, and in all instances the owners reported that they had not observed any recurrence of lameness.

\section{Discussion}

The use of a temporary transarticular stabilization with a locking plate has previously been described in a dog affected by medial shoulder luxation. ${ }^{25}$ It was our objective to determine whether that technique could be modified to provide adequate immobilization of the tarsocrural joint during the initial phase of tendon healing.

Our case series demonstrates that a temporary transarticular calcaneo-tibial stabilization with a locking plate maintains the tibiotarsal joint in extension for 6 weeks to allow offloading of the common calcaneal tendon, is associated with relatively low morbidity in small cohort of dogs and leads to a satisfactory functional outcome. All four hunting dogs returned to hunting activity with subjectively assessed full function of the affected limb. Most of the cases were presented acutely, with the injury having occurred while the dogs were hunting and five out of nine cases also had a concomitant wound. All tendon ends were directly sutured without the need for augmentation techniques. ${ }^{10,26}$ All tendons healed and no complications at the level of the tenorrhaphy were noted in the postoperative period. With medial locking plate application, adequate visibility of the caudal calcaneal cortex is mandatory for precise locking screw insertion, cutting through and elevating the caudodistal part of the superficial digital flexor retinaculum with- out lateral dislocation of the superficial digital flexor tendon. The caudoproximal part of the retinaculum should be preserved to reduce the risk of tendon luxation in the postoperative period. $^{24}$

Most of the locking plates used in this study were ' $\mathrm{T}$ '- or 'L'-shaped plates and we noticed that the 'L' plates allow easier plate contouring and positioning. We recommend that the surgeon has a range of options available intraoperatively to choose the implant that better fits with the fully extended hock joint.

In most of our cases, we selected implants of 1.5 or $2.0 \mathrm{~mm}$ thickness. Thin plates allow the surgeon to perform the complex anatomical plate contouring around the medial malleolus that is necessary for correct plate application. Moreover, the locking plate is applied on the tension side of the hock joint and seemed to be adequate in resisting to the tensile forces applied. ${ }^{27}$

Despite the weight limit suggested by the company based on a published study, ${ }^{28}$ the 1.9 to $2.5 \mathrm{~mm}$ MINI system was applied in dogs up to $15 \mathrm{~kg}$ of weight and the 3.0 to $3.5 \mathrm{~mm}$ LARGE system was applied in dogs more than $15 \mathrm{~kg}$. To reduce the risk of iatrogenic fractures, we suggest insertion of $3.0 \mathrm{~mm}$ screws in the calcaneus of dogs ranging in weight between 15 and $20 \mathrm{~kg}$; however, this requires further clinical investigation.

The locking mechanism allows placement of a plate at a given distance to the bone, but a plate-bone distance less than $2 \mathrm{~mm}$ is recommended to reduce bending forces over the screw neck and to increase the fixation stiffness. ${ }^{29}$

Screw loosening is less likely to occur than with conventional plating, and resistance to displacement is greater 
when only a few screws are used. ${ }^{28}$ In our case series, screw pull out occurred in one case (case 6 ) and we speculate that it was related to an excessive plate contouring that leads to a failure of the conical coupling between the screw head and the bushing during screw tightening. We recommend therefore using the company's plate benders to protect the titanium bushings of the stainless-steel plate.

We suggest inserting the screws as close as possible to the caudal cortex of the calcaneus to preserve enough bone stock and reduce the risk of avulsion fractures. Attention must be paid during screw insertion due to the converging direction of the two tibial screws. Accurate plate contouring leads ${ }^{20}$ to a precise locking screw insertion and reduces the potential risk of postoperative tibial or calcaneal fractures.

It is important that a tendon anastomosis is not subjected to tensile forces exceeding the limits of the repair site strength during the early postoperative phase since this stress can lead to gap formation or repair failure, accordingly to primary tendon repair method used. Tendons with a gap $>3 \mathrm{~mm}$ heal at a slower rate and have a decreased ultimate tensile resistance at 6 weeks after repair, compared with results for tendons healing with no gap or a gap $<3 \mathrm{~mm} .^{5}$

In the present study, joints were immobilized for 6 weeks, as recommended by Braden. ${ }^{30}$ Studies have shown that repaired tendons regained only $56 \%$ of normal breaking strain by 6 weeks, and $79 \%$ of normal breaking strain after 1 year. ${ }^{31}$ Longer rest of an injured tendon can be counterproductive, as some loading of the tendon is required to stimulate repair and appropriate remodelling of collagen fibers. ${ }^{32}$ Optimum tendon healing requires some degree of strain along the length of the tendon to stimulate proper recovery because the strength of collagenous tissue is positively affected by exercise and lessened with immobilization. ${ }^{33}$

Different tibiotarsal immobilization methods following common calcaneal tendon repair have been described. ${ }^{4,6-8,16-18}$ Most complications after tenorrhaphy are considered to be related to the immobilization technique rather than to the method of tendon repair. ${ }^{23,34}$ Despite the high incidence of open injuries, no significant effect on complication rate or outcome was noted with the use of an internal fixation technique for temporary tarsocrural joint immobilization.

No major complications were observed. Minor complications occurred in two cases (case 1, case 6), osteomyelitis and screw pull-out ( - Fig. 5). A complication rate of up to $35 \%$ has been described by Corr and colleagues in 38 dogs treated with calcaneo-tibial positional screws and external coaptation. ${ }^{4}$ Cast application with or without other stabilization methods often results in minor complications such as skin lesions and infection. ${ }^{23}$ Meeson and colleagues ${ }^{21}$ reported the development of soft-tissue injuries in $63 \%$ of cases, $20 \%$ of which were severe.

The incidence of postoperative minor complications in our case series may be lower than previously reported due to the castless management of the postoperative phase, but further cases are required to see if this result is reproduced in a bigger cohort. In Corr's study, ${ }^{4}$ implant-related major complications occurred in $8 \%$ of cases and required screw replacement. In our case series, no major complications occurred and no skin lesions were reported during postoperative evaluations.

As described, this method of temporary stabilization incurs the additional costs of four locking screws and a Fixin plate, which is significantly higher than the cost of a single calcaneo-tibial screw or cast application. A dedicated implant could significantly reduce surgical time, while helping the surgeon that is learning the technique to insert the two calcaneal locking screws with the optimal direction.

There are several limitations to this study, mostly deriving from its retrospective design. The small number of cases limits the significance of the study and makes it difficult to draw broad conclusions about the incidence of complications.

Prospective studies are preferred, but are logistically difficult particularly with a relatively uncommon injury. Assessment of tendon healing judgement was mostly based on clinical palpation as ultrasound examination was not performed in all cases. There was no objective outcome evaluation such as goniometric range of motion measurements before and after temporary hock joint immobilization or gait analyses to assess the effects of such a stiff temporary fixation on joint mobility and cartilage health. Clinical functional outcome was judged in all the cases by the surgeon and may have introduced bias. Although the 12-week outcome of all the eight cases was favourable, a limitation of our study was that long-term follow-up relied on owner observations. Future biomechanical studies, to evaluate the strength of the temporary internal fixation technique and to compare it with other reported techniques, would be desirable.

In conclusion, the use of a transarticular locking plate for temporary immobilization of the tarsocrural joint after surgical repair of common calcaneal tendon rupture appears to be an effective technique with comparable results to other reported techniques. Further focused evaluation is warranted to evaluate calcaneo-tibial locking plate fixation through a prospective clinical trial with assessment on long-term outcome and occurrence of complications in a larger number of dogs.

\section{Author's Contributions}

A.B.B., F.C.F. and L.A.P. contributed to the conception of the study, study design, acquisition of data, data analysis and interpretation, and drafting/revising and approving the submitted manuscript. L.O.A., M.S., B.V., B.P. and L.A.P. acquired the data, performed data analysis and interpretation, and drafted/revised and approved the submitted manuscript.

\section{Conflict of Interest}

All the authors report no financial support from Intrauma S.p.a. for the submitted work. A.B.B, L.O, B.P and L.A.P are speakers for Intrauma courses. All others authors have no conflicts of interest. 


\section{Acknowledgments}

The authors would like to thank Maria Cristina Boero Baroncelli for surgical approach's drawing and Danielle Wells for grammar correction.

\section{References}

1 Carmichael S, Marshall W. Tarsus and metatarsus. In: Tobias KM, Johnston SA, eds. Veterinary Surgery: Small Animal. 1st edition St. Louis: Saunders Elsevier; 2012:1023-1028

2 Reinke J. Treatment of avulsion of the gastrocnemius tendon. In: Bojrab MJ, Ellison G, Slocum G, eds. Current Techniques in Small Animal Surgery. 4th edition Baltimore: Williams and Wilkins; 1988:1257-1260

3 Moores AP, Comerford EJ, Tarlton JF, Owen MR. Biomechanical and clinical evaluation of a modified 3-loop pulley suture pattern for reattachment of canine tendons to bone. Vet Surg 2004;33(04): 391-397

4 Corr SA, Draffan D, Kulendra E, Carmichael S, Brodbelt D. Retrospective study of Achilles mechanism disruption in 45 dogs. Vet Rec 2010;167(11):407-411

5 Gelberman RH, Boyer MI, Brodt MD, Winters SC, Silva MJ. The effect of gap formation at the repair site on the strength and excursion of intrasynovial flexor tendons. An experimental study on the early stages of tendon-healing in dogs. J Bone Joint Surg Am 1999;81(07):975-982

6 Morshead D, Leeds EB. Kirschner-Ehmer apparatus immobilization following Achilles tendon repair in six dogs. Vet Surg 1984; 13:11-14

7 De Haan JJ, Goring RL, Renberg C, Bertrand S. Modified transarticular external skeletal fixation for support of Achilles tenorrhaphy in four dogs. Vet Comp Orthop Traumatol 1995;8:32-35

8 Guerin S, Burbidge H, Firth E, Fox S. Achilles tenorrhaphy in five dogs: a modified surgical technique and evaluation of a cranial half cast. Vet Comp Orthop Traumatol 1998;11:205-210

9 Vaughan LC. The use of carbon fibre implants for the repair of Achilles tendon rupture in dogs. J Small Anim Pract 1981;22(10): 629-634

10 Nielsen C, Pluhar GE. Outcome following surgical repair of Achilles tendon rupture and comparison between postoperative tibiotarsal immobilization methods in dogs: 28 cases (1997-2004). Vet Comp Orthop Traumatol 2006;19(04):246-249

11 Gall TT, Santoni BG, Egger EL, Puttlitz CM, Rooney MB. In vitro biomechanical comparison of polypropylene mesh, modified threeloop pulley suture pattern, and a combination for repair of distal canine Achilles' tendon injuries. Vet Surg 2009;38(07):845-851

12 Putterman AB, Duffy DJ, Kersh ME, Rahman H, Moore GE. Effect of a continuous epitendinous suture as adjunct to three-loop pulley and locking-loop patterns for flexor tendon repair in a canine model. Vet Surg 2019;48(07):1229-1236

13 Cocca CJ, Duffy DJ, Kersh ME, Kim W, Groenewold A, Moore GE. Biomechanical comparison of three epitendinous suture patterns as adjuncts to a core locking loop suture for repair of canine flexor tendon injuries. Vet Surg 2019;48(07):1245-1252

14 Duffy DJ, Curcillo CP, Chang YJ, Gaffney L, Fisher MB, Moore GE. Biomechanical evaluation of an autologous flexor digitorum lateralis graft to augment the surgical repair of gastrocnemius tendon laceration in a canine ex vivo model. Vet Surg 2020;49(08):1545-1554

15 Zellner EM, Hale MJ, Kraus KH. Application of tendon plating to manage failed calcaneal tendon repairs in a dog. Vet Surg 2018;47 (03):439-444
16 Reinke JD, Mughannam AJ, Owens JM. Avulsion of the gastrocnemius tendon in 11 dogs. JAAHA 1993;29:410-418

17 Norton J, Decamp C, Yu J, Rooks R. Use of a single-ring transarticular fixator construct for immobilisation of the talocrural joint following common calcaneal tenorrhaphy. Vet Comp Orthop Traumatol 2009;22(05):430-435

18 Mich PM. The emerging role of veterinary orthotics and prosthetics (V-OP) in small animal rehabilitation and pain management. Top Companion Anim Med 2014;29(01):10-19

19 Petazzoni M, Urizzi A, Verdonck B, Jaeger G. Fixin internal fixator: concept and technique. Vet Comp Orthop Traumatol 2010;23(04): 250-253

20 Berg RJ, Egger EL. In vitro comparison of the three loop pulley and locking loop suture patterns for repair of canine weight-bearing tendons and collateral ligaments. Vet Surg 1986;15:107-110

21 Meeson RL, Davidson C, Arthurs GI. Soft-tissue injuries associated with cast application for distal limb orthopaedic conditions. A retrospective study of sixty dogs and cats. Vet Comp Orthop Traumatol 2011;24(02):126-131

22 Anderson GM, Lewis DD, Radasch RM, Marcellin-Little DJ, Degna MT, Cross AR. Circular external skeletal fixation stabilization of antebrachial and crural fractures in 25 dogs. J Am Anim Hosp Assoc 2003;39(05):479-498

23 Reinke JD, Mughannam AJ. Lateral luxation of the superficial digital flexor tendon in 12 dogs. J Am Anim Hosp Assoc 1993; 29:303-309

24 Post C, Guerrero T, Voss K, Montavon PM. Temporary transarticular stabilization with a locking plate for medial shoulder luxation in a dog. Vet Comp Orthop Traumatol 2008;21(02):166-170

25 Shani J, Shahar R. Repair of chronic complete traumatic rupture of the common calcaneal tendon in a dog, using a fascia lata graft. Case report and literature review. Vet Comp Orthop Traumatol 2000;13:104-108

26 Morton MA, Thomson DG, Rayward RM, Jiménez-Peláez M, Whitelock RG. Repair of chronic rupture of the insertion of the gastrocnemius tendon in the dog using a polyethylene terephthalate implant. Early clinical experience and outcome. Vet Comp Orthop Traumatol 2015;28(04):282-287

27 Prasad K, Bazaka O, Chua M, et al. Metallic biomaterials: current challenges and opportunities. Materials (Basel) 2017;10(08): $1-33$

28 Nicetto T, Petazzoni M, Urizzi A, Isola M. Experiences using the Fixin locking plate system for the stabilization of appendicular fractures in dogs: a clinical and radiographic retrospective assessment. Vet Comp Orthop Traumatol 2013;26(01):61-68

29 Rotne R, Bertollo N, Walsh W, Dhand NK, Voss K, Johnson KA. Influence of plate-bone contact on cyclically loaded conically coupled locking plate failure. Injury 2014;45(03):515-521

30 Braden TD. Musculotendinous rupture of the Achilles apparatus and repair using internal fixation only. Vet Med Small Anim Clin 1974;69(06):729-735

31 Dueland R, Quenin J. Triceps tenotomy: biomechanical assessment of healing strength. J Am Anim Hosp Assoc 1980;16:507-512

32 Mason ML, Allen HS. The rate of healing of tendons: an experimental study of tensile strength. Ann Surg 1941;113(03): 424-459

33 Lister SA, Renberg WC, Roush JK. Efficacy of immobilization of the tarsal joint to alleviate strain on the common calcaneal tendon in dogs. Am J Vet Res 2009;70(01):134-140

34 Sugiyama T, Woodward A, Ryan SD. Biomechanical evaluation of 6 transarticular tibiotarsal immobilization methods in canine cadaveric limbs. Vet Surg 2018;47(05):705-714 